Published in final edited form as:

J Am Geriatr Soc. 2019 March ; 67(3): 553-557. doi:10.1111/jgs.15708.

\title{
Relationship between Functional Improvement and Cognition in Short-Stay Nursing Home Residents
}

\author{
Lacey Loomer, $\mathrm{MSPH}^{\star}$, Brian Downer, $\mathrm{PhD}^{\dagger}$, and Kali S. Thomas, PhD, MA ${ }^{*}, \ddagger$ \\ "Department of Health Services, Policy and Practice, School of Public Health, Brown University, \\ Providence, Rhode Island; tDivision of Rehabilitation Sciences, University of Texas Medical \\ Branch, Galveston, Texas $\ddagger$ Department of Veterans Affairs Medical Center, Providence, Rhode \\ Island.
}

\begin{abstract}
OBJECTIVES: Improving function is an important outcome of postacute care in skilled nursing facilities (SNFs), but cognitive impairment can limit a resident's ability to improve during a postacute care stay. Our objective was to examine the association between residents' cognitive status on admission and change in self-care and mobility during a Medicare-covered SNF stay.
\end{abstract}

DESIGN: Retrospective analysis of Medicare beneficiaries who had a new SNF stay between January and June 2017.

SETTING: SNFs in the United States.

PARTICIPANTS: Newly admitted residents with Medicare-covered SNF stays between January and June $2017(\mathrm{n}=246$ 395).

MEASUREMENTS: Residents' self-care and mobility at SNF admission and discharge were determined using items from Section GG (eating, oral hygiene, toileting hygiene, sit to lying, lying to sitting, sit to stand, chair/bed transfer, and toilet transfer) of the Minimum Data Set. Residents were classified as cognitively intact, mildly impaired, moderately impaired, or severely impaired, according to the Cognitive Function Scale. Multivariable regression models controlling for residents' demographic and clinical characteristics and SNF fixed effects were used to identify residents whose discharge scores for self-care and mobility were better or the same as expected according to their cognitive status on admission.

RESULTS: Residents who were cognitively impaired on admission had lower functional status on admission and were less likely to improve in self-care and mobility compared with residents who were cognitively intact. Approximately $63 \%$ of residents who were cognitively intact had

Address correspondence to Lacey Loomer, MSPH, Department of Health Services, Policy and Practice, Brown University School of Public Health, 121 South Main Street, G-121-3, Providence, RI 02912; lacey_loomer@brown.edu.

Author Contributions: Study concept and design: Loomer, Downer, and Thomas. Acquisition of data: Thomas. Analysis and interpretation of data: Loomer, Downer, and Thomas. Preparation of the manuscript: Loomer, Downer, and Thomas.

This article was previously presented as a poster at the 2018 Academy Health Annual Research Meeting.

Conflict of Interest: The authors have no conflicts of interest to disclose. 
discharge scores for self-care and mobility that were better or the same as expected compared with $45 \%$ of residents with severe cognitive impairment.

CONCLUSIONS: Cognitive impairment is associated with poorer self-care and mobility function among SNF residents. These findings have important implications for clinicians, who may need additional support when caring for residents with cognitive impairment to make the same improvements in functional status as residents who are cognitively intact.

\section{Keywords}

nursing homes; functional status; quality measures; cognitive impairment

Skilled nursing facilities (SNFs) are the most common site for postacute care (PAC) among Medicare beneficiaries, ${ }^{1}$ accounting for nearly $50 \%$ of Medicare's PAC spending in $2015 .{ }^{2}$ Older adults with cognitive impairment are commonly discharged to an SNF for PAC. ${ }^{3-5}$ Improving residents' functional status is an important outcome of PAC, but cognitive impairment can limit a resident's ability to regain function. 6,7

The Resident Assessment Instrument Minimum Data Set (MDS) is used to evaluate nursing home $(\mathrm{NH})$ residents' functional status, health, and treatment needs, and to develop personspecific care plans. MDS data are also used to derive publicly reported quality measures (QMs). New self-care and mobility items were added to the MDS by the Centers for Medicare \& Medicaid Services in October $2016^{8}$ so that functional measures can be comparable across different PAC settings. These new items will be used to calculate QMs for change in self-care and mobility during a SNF stay and level of functioning for self-care and mobility at SNF discharge. ${ }^{9}$ Public reporting of these QMs will begin in 2020, and SNFs will be financially responsible for their performance on these measures as part of the SNF Quality Reporting Program. ${ }^{9}$

The impact of cognitive impairment on the improvement of functional status was studied in PAC settings. $.67,10,11$ However, most studies used functional status measures not included in the MDS. ${ }^{7,10}$ Furthermore, no studies examined how SNF residents with cognitive impairment perform on the new self-care and mobility MDS items. Our objective was to examine changes in SNF residents' self-care and mobility using the new MDS items and to identify differences according to cognitive status at admission.

\section{METHODS}

The analytic sample includes Medicare Part A beneficiaries who had a Prospective Payment System (PPS) stay between January 1, 2017, and June 30, 2017, but had not been in a NH in 2016. Following the technical documentation for calculating the new QMs, ${ }^{12}$ we excluded residents who (1) had incomplete information on any independent variables, (2) had different Medicare stay and facility entry dates, (3) were in the NH less than 3 days; (4) were younger than 21 years, (5) did not receive any physical or occupational therapy, (6) were comatose, (7) were discharged to hospice or died, or (8) had an unplanned discharge. The final analytic sample included 246,395 SNF PPS stays. 


\section{Self-care and mobility measures}

Measures for self-care and mobility came from Section GG of the MDS 3.0 v1.14.1.

Detailed information for how the Section GG items are to be completed is provided in the MDS Resident Assessment Instrument Manual. ${ }^{13}$ The self-care measures included eating, oral hygiene, and toileting hygiene. The mobility measures included moving from a sitting to lying position, moving from a lying to a sitting position, sit to stand, chair/bed- to-chair transfer, and toilet transfer. These items are scored on a scale of 1 (dependent: helper does all of the effort) to 6 (independent: resident completes the activity independently with no assistance from a helper). We calculated composite measures for self-care and mobility by summing the individual items.

\section{Classifying cognitive status}

The MDS v.3.0 includes performance-based and staff observation measures for cognitive functioning. The Cognitive Function Scale is a validated measure that integrates information from the Brief Interview of Mental Status ${ }^{14,15}$ and Cognitive Performance Scale ${ }^{16}$ to classify residents as cognitively intact, mildly impaired, moderately impaired, or severely impaired. 17

\section{Improvement in self-care and mobility}

We calculated improvement by subtracting residents' admission score from their discharge score. We calculated change scores for the individual items and the composite measures. The outcome of interest was the percentage of residents who improved or stayed the same.

\section{Expected self-care and mobility}

Residents' expected discharge scores for self-care and mobility were calculated following the technical documentation developed by RTI International. ${ }^{12}$ We used a linear multivariable regression model that included NH fixed effects and controlled for a variety of resident-level variables: age, admission self-care and mobility function scores as linear and squared terms, and measures that may impact residents' self-care and mobility (see next section on independent variables). Next, we subtracted the observed discharge scores from the expected discharge scores, and created a dichotomous variable to identify residents whose scores at discharge were the same or higher than expected. The outcome of interest was the percentage of residents whose scores at discharge were the same or higher than expected.

\section{Independent variables}

Independent variables were assessed using the MDS and were chosen based on the covariates that will be in the risk adjustment process for the QMs. ${ }^{12}$ Age at admission was categorized as younger than 65,65 to 74 (reference), 75 to 84,85 to 90 , and 90 years and older. We also included admission diagnoses including stroke, cancer, Alzheimer's disease/ dementia, hip or other fracture, multiple sclerosis, Huntington's disease, Parkinson's disease, psychiatric mood disorder (depression, manic depression, psychotic disorder, or schizophrenia), coronary artery disease, diabetes, and infections. Measures of communication included residents' ability to make themselves understood and to understand 
others. A resident was considered continent (urinary and bowel) if they were always continent according to Section $\mathrm{H}$. We included indicators if a resident had any stage 2 or 3 pressure ulcers present and if a resident had a history of falls in the last 6 months.

\section{Statistical analyses}

We used analysis of variance (ANOVA) to assess differences according to cognitive status at admission for three measures of functional status: average admission and discharge scores; percentage of residents who improved or stayed the same from admission to discharge; and percentage of residents whose observed discharge score was higher or the same as their expected score as calculated by the multivariate regression models. Next, we used $t$ tests to compare these outcomes between successive levels of cognitive impairment (intact to mild, mild to moderate, and moderate to severe). A $p$-value $<.001$ was considered statistically significant. All analyses were completed using SAS v.9.4 and Stata 14.0 software. The study was reviewed and approved by the Brown University institutional review board.

\section{RESULTS}

The average length of stay was 24 days, and most residents were between 65 and 84 years of age (Supporting Information Table S1). Overall, $68 \%$ of residents had no cognitive impairment at admission, $18.3 \%$ had mild impairment, $11.8 \%$ had moderate impairment, and $1.7 \%$ had severe impairment. About $20 \%$ of the sample had a fracture, $30 \%$ had diabetes, and $27 \%$ had psychiatric mood disorders. Almost half of the sample experienced some urinary incontinence, and half had fallen in the last 6 months.

Figure 1 displays the individual items for self-care and mobility at admission and discharge according to cognitive status at admission. ANOVA tests showed significant differences in admission and discharge scores for all items according to cognitive status. Residents with severe cognitive impairment had functional status scores that were 2 points lower than those who were cognitively intact. The discharge scores for residents with severe cognitive impairment were less than 1 point higher than the admission scores, compared with 2 points higher for residents who were cognitively intact.

A total of $97 \%$ of residents who were cognitively intact improved in the mobility composite score compared with $95 \%, 92 \%$, and $87 \%$ for residents with mild, moderate, and severe cognitive impairment, respectively (Table 1). ANOVA results showed significant differences in the percentage of residents that improved or maintained the same level of function according to cognitive status. No statistically significant differences were found between residents with moderate and severe cognitive impairment for eating and toileting hygiene items.

There were significant differences according to cognitive status in the percentage of residents whose observed discharge score met or exceed their expected score (Table 2). Over $60 \%$ of residents who were cognitively intact at admission had observed discharge scores for each self-care and mobility item that met or exceeded the expected scores compared with less than $50 \%$ of residents with severe cognitive impairment. In total, $79 \%$ of residents who were cognitively intact had an observed discharge score that met or exceeded the expected 
score compared with $64 \%, 54 \%$, and $50 \%$ of residents with mild, moderate, and severe cognitive impairment, respectively. There were significant differences for all mobility items in the percentage of residents whose observed discharge score met or exceeded their expected score between residents with mild and moderate cognitive impairment. The only significant differences between the moderate and severe impairment groups were for the composite mobility score and for toilet transfer.

\section{DISCUSSION}

Our objective was to examine changes in SNF residents' self-care and mobility according to cognitive status at admission. The percentage of residents whose observed self-care and mobility scores improved or stayed the same and who had discharge scores that were the same or higher than expected were substantially lower for residents with cognitive impairment of any severity. The greatest differences were between residents who were cognitively intact and those who were severely impaired. It is not surprising that residents with cognitive impairment showed less improvement in self-care and mobility, but the large magnitude of these differences make our findings important.

Our findings have several clinical implications for the rehabilitation of SNF residents with cognitive impairment. We found that nearly $85 \%$ of residents with moderate or severe cognitive impairment improved or stayed the same in self-care and mobility during a SNF stay, compared with $95 \%$ for residents with no cognitive improvement. The difference between cognitive impairment is consistent with Barnes et al, who found higher cognitive function on admission was associated with large gains in functional status. ${ }^{10}$ Overall, the proportion improving or maintaining functional status is higher compared with Wysocki et al, who found $60 \%$ of short-stay residents who improved in activities of daily living, ${ }^{6}$ but this is likely due to the inclusion of maintaining functional status in our measure. Our study provides evidence that individuals with cognitive impairment can regain function during a SNF stay. However, our analyses revealed that only $50 \%$ of residents with moderate or severe impairment had self-care and mobility scores at discharge that were equal to or higher than expected. This indicates that residents with cognitive impairment may require more intense therapy before meaningful gains in function are observed. ${ }^{10}$ The rehabilitation of individuals with cognitive impairment can be complicated by behavioral, neuropsychiatric, and cognitive symptoms commonly associated with dementia. ${ }^{18-20}$ Consequently, therapists may have to use novel strategies and make specific accommodations for the rehabilitation services to be most effective. ${ }^{19,21-23}$

Starting in 2020, SNFs will be required to report publicly the QMs based on these new items. We identified that residents with significant cognitive impairment performed significantly worse on these outcomes than residents with moderate cognitive impairment. This is important because the QM technical documentation combines residents classified as moderately and severely impaired into a single impairment category. Thus the QMs may not adequately account for the potentially higher risk of worse functional outcomes for residents with severe cognitive impairment. A consequence is NHs that admit a high percentage of residents with severe cognitive impairment may be unfairly penalized on these QMs. 
This study relies on administrative data and is therefore subject to the limitations therein. ${ }^{24}$ As an additional limitation, we were not able to include the full list of Section GG items (ie, showering, upper and lower body dressing, putting on/taking off footwear, walking on an uneven surface, walking up/down steps, and picking up an object) or adjust for the full set of covariates in the technical documentation (ie, $\mathrm{NH}$ residents with dementia can have substantial difficulty dressing themselves without assistance ${ }^{25}$ and cognitive impairment contributes to slow gait ${ }^{26}$ and poor balance). ${ }^{27}$ This makes it likely that SNF residents with moderate or severe cognitive impairment will have worse performance on the additional items than residents who are cognitively intact. Consequently, differences in the composite measures may be even larger than what we detected using the limited item set. Lastly, this study classifies improvement as any discharge score higher than the admission score; however, it will be important to establish a minimally clinically important difference for these items to better inform clinical practice.

In conclusion, this study provides an initial summary of the new functional status items and how cognitive impairment impacts functional improvement during a SNF stay. Residents with higher cognitive impairment exhibited less improvement on all items compared with residents who were cognitively intact on admission. Our findings have implications for clinicians because SNF residents with cognitive impairment may need additional support and more intensive rehabilitation to make the same functional gains as residents who are cognitively intact.

\section{Supplementary Material}

Refer to Web version on PubMed Central for supplementary material.

\section{ACKNOWLEDGMENTS}

Financial Disclosure: This study was funded by P01A G027296 from the US National Institute on Aging. Ms. Loomer's time is supported by NIH-NIA 2T32AG023482-13. Dr. Downer's time is covered by the University of Texas Medical Branch Pepper Center (NIA P30AG024832-14). Dr. Thomas's time is covered by a Career Development Award from the Veterans Health Administration (CDA14-422).

Sponsor's Role: Funding sources did not have any role in the design, methods, subject recruitment, data collections, analysis, or preparation of the article.

\section{REFERENCES}

1. Tian W An all-payer view of hospital discharge to postacute care, 2013. HCUP Statistical Brief \#205 Rockville, MD: Agency for Healthcare Research and Quality, 2016.

2. Medicare Payment Advisory Commission. A Data Book: Health Care Spending and the Medicare Program Washington, DC: MedPAC, 2017.

3. Bell SP, Vasilevskis EE, Saraf AA et al. Geriatric syndromes in hospitalized older adults discharged to skilled nursing facilities. J Am Geriatr Soc 2016; 64:715-722. [PubMed: 27059831]

4. Callahan CM, Tu W, Unroe KT, LaMantia MA, Stump TE, Clark DO. Transitions in care in a nationally representative sample of older Americans with dementia. J Am Geriatr Soc 2015;63:1495-1502. [PubMed: 26200764]

5. Givens JL, Mitchell SL, Kuo S, Gozalo P, Mor V, Teno J. Skilled nursing facility admissions of nursing home residents with advanced dementia. J Am Geriatr Soc 2013;61:1645-1650. [PubMed: 24117283] 
6. Wysocki A, Thomas KS, Mor V. Functional improvement among short-stay nursing home residents in the MDS 3.0. J Am Med Dir Assoc 2015;16: 470-474. [PubMed: 25659622]

7. Cary MP, Pan W, Sloane R et al. Self-care and mobility following postacute rehabilitation for older adults with hip fracture: a multilevel analysis. Arch Phys Med Rehabil 2016;97:760-771. [PubMed: 26836951]

8. RTI International. Skilled Nursing Facility Quality Reporting Dental Program: Quality Measures Specifications for FY 2016 Notice of Proposed Rulemaking https://www.federalregister.gov/ documents/2015/08/04/2015-18950/medicare-program-prospective-payment-system-andconsolidated-billing-forskilled-nursing-facilities

9. Centers for Medicare and Medicaid Services. Skilled Nursing Facility Quality Reporting Program (SNF QRP): Requirements for the Fiscal Year (FY) 2020 Program Year https://www.cms.gov/ Medicare/Quality-Initiatives-Patient-Assessment-Instruments/NursingHomeQualityInits/ Downloads/SNFQRP-Requirements-for-the-Fiscal-Year-FY2020-Program-Year.pdf

10. Barnes C, Conner D, Legault L, Reznickova N, Harrison-Felix C. Rehabilitation outcomes in cognitively impaired patients admitted to skilled nursing facilities from the community. Arch Phys Med Rehabil 2004;85: 1602-1607. [PubMed: 15468018]

11. Yu F, Evans LK, Sullivan-Marx EM. Functional outcomes for older adults with cognitive impairment in a comprehensive outpatient rehabilitation facility. J Am Geriatr Soc 2005;53:1599_ 1606. [PubMed: 16137294]

12. RTI International. Final specifications for SNF QRP quality measures and standardized resident assessment data elements 2017 https://www.cms.gov/Medicare/Quality-Initiatives-PatientAssessment-Instruments/NursingHomeQualityInits/Downloads/Final-Specifications-for-SNFQRP-Quality-Measures-and-Standardized-Resident-Assessment-Data-Elements-EffectiveOctober-1-2018.pdf

13. Centers for Medicare and Medicaid Services. MDS 3.0 RAI Manual v.1.14 2016 https:// www.cms.gov/Medicare/Quality-Initiatives-Patient-Assessment-Instruments/ NursingHomeQualityInits/NHQIMDS30-ArchivedRAIManuals.html

14. Saliba D, Buchanan J, Edelen MO et al. MDS 3.0: Brief interview for mental status. J Am Med Dir Assoc 2012;13:611-617. [PubMed: 22796362]

15. Chodosh J, Edelen MO, Buchanan JL et al. Nursing home assessment of cognitive impairment: Development and testing of a brief instrument of mental status. J Am Geriatr Soc 2008;56:20692075. [PubMed: 19016941]

16. Morris JN, Fries BE, Mehr DR et al. MDS cognitive performance scale. J Gerontol 1994;49:M174-M182. [PubMed: 8014392]

17. Thomas KS, Dosa D, Wysocki A, Mor V. The minimum data set 3.0 cognitive function scale. Med Care 2017;55:e68-e72. [PubMed: 25763665]

18. Gialanella B, Prometti P, Monguzzi V, Ferlucci C. Neuropsychiatric symptoms and rehabilitation outcomes in patients with hip fracture. Am J Phys Med Rehabil 2014;93:562-569. [PubMed: 24508934]

19. McGilton K, Weels J, Teare G et al. Rehabilitating patients with dementia who have had a hip fracture. Part I: Behavioral symptoms that influence care. Top Geriatr Rehabil 2007;23:161-173.

20. Galik EM, Resnick B, Pretzer-Aboff I. 'Knowing what makes them tick': Motivating cognitively impaired older adults to participate in restorative care. Int J Nurs Pract 2009;15:48-55. [PubMed: 19187169]

21. McGilton K, Wells J, Davis A et al. Rehabilitating patients with dementia who have had a hip fracture. Part II: Cognitive symptoms that influence care. Top Geriatr Rehabil 2007;23:174-182.

22. Resnick B, Gruber-Baldini AL, Zimmerman S et al. Nursing home resident outcomes from the rescare intervention. J Am Geriatr Soc 2009;57: 1156-1165. [PubMed: 19570158]

23. Galik E, Resnick B, Hammersla M, Brightwater J. Optimizing function and physical activity among nursing home residents with dementia: Testing the impact of function-focused care. Gerontologist 2014;54:930-943. [PubMed: 24092822]

24. Rahman AN, Schnelle JF. The nursing home culture-change movement: Recent past, present, and future directions for research. Gerontologist 2008; 48:142-148. [PubMed: 18483426] 
25. Cohen-Mansfield J, Creedon MA, Malone T, Parpura-Gill A, Dakheel-Ali M, Heasly C. Dressing of cognitively impaired nursing home residents: description and analysis. Gerontologist 2006;46:89-96. [PubMed: 16452288]

26. Amboni M, Barone P, Hausdorff JM. Cognitive contributions to gait and falls: Evidence and implications. Mov Disord 2013;28:1520-1533. [PubMed: 24132840]

27. Tangen GG, Engedal K, Bergland A, Moger TA, Mengshoel AM. Relationships between balance and cognition in patients with subjective cognitive impairment, mild cognitive impairment, and Alzheimer disease. Phys Ther 2014;94:1123-1134. [PubMed: 24764071] 


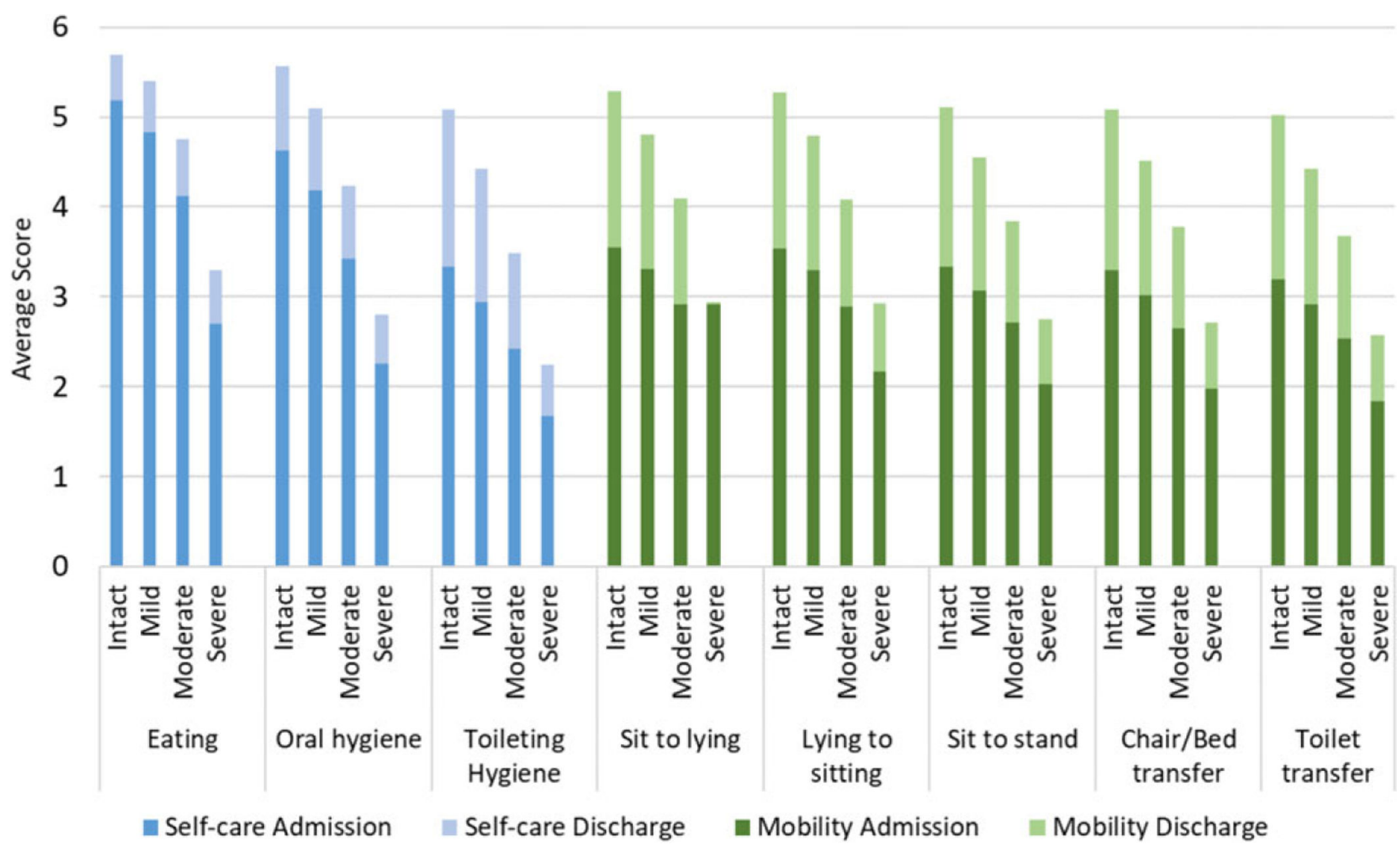

Figure 1.

Average observed self-care and mobility scores on admission and discharge, by cognitive status at admission Scores ranged from 1 (dependent) to 6 (independent). Means for each item were compared across groups of the Cognitive Function Scale and were significantly different as determined by analysis of variance for all items $(p<.001)$. 


\section{Table 1}

Percentage of residents whose observed self-care and mobility performance improved or stayed the same between admission and discharge ${ }^{a}$

\begin{tabular}{lcccc}
\hline \multicolumn{1}{c}{ Mean (SD) } & Intact & Mild & Moderate & Severe \\
\hline Self-Care items & & & & \\
Eating & 95.9 & $92.7^{b}$ & $88.5^{c}$ & 86.8 \\
Oral hygiene & 95.8 & $96.7^{b}$ & $88.7^{c}$ & $86.6^{c}$ \\
Toileting hygiene & 96.9 & $95.0^{b}$ & $92.7^{c}$ & 92.0 \\
Composite self-care score & 95.5 & $92.1^{b}$ & $87.2^{c}$ & $84.3^{d}$ \\
Mobility items & & & & \\
Sit to lying & 97.4 & $95.7^{b}$ & $93.5^{c}$ & $91.1^{d}$ \\
Lying to sitting & 97.4 & $95.7^{b}$ & $93.5^{c}$ & $90.8^{d}$ \\
Sit to stand & 98.0 & $96.5^{b}$ & $94.1^{c}$ & $91.8^{d}$ \\
Chair/Bed-to-chair transfer & 98.1 & $96.7^{b}$ & $94.4^{c}$ & $92.6^{d}$ \\
Toilet transfer & 97.8 & $96.3^{b}$ & $94.1^{c}$ & $92.5^{d}$ \\
Composite mobility score & 97.0 & $94.8^{b}$ & $91.5^{c}$ & $87.6^{d}$ \\
\hline
\end{tabular}

${ }^{a}$ By cognitive status at admission $(\mathrm{N}=246,395)$.

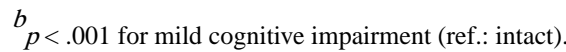

$c_{p}^{c} .001$ for moderate cognitive impairment (ref.: mild).

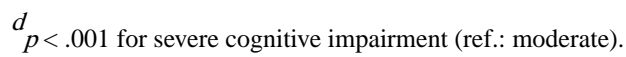

Notes: Means of the percentage of residents whose observed score improved or stayed the same from admission to discharge for each item were compared across groups of Cognitive Function Scale (CFS) and were significantly different as determined by analysis of variance for all items $(p$ $<.001)$. The $t$ tests were used to compare means of items between groups of CFS. 


\section{Table 2}

Percentage of residents whose observed self-care and mobility scores at discharge are the same or higher than their expected discharge score ${ }^{a}$

\begin{tabular}{lcccc}
\hline \multicolumn{1}{c}{ Mean (SD) } & Intact & Mild & Moderate & Severe \\
\hline Self-care items & & & & \\
Eating & 78.8 & $64.3^{b}$ & $54.0^{c}$ & $50.0^{d}$ \\
Oral hygiene & 72.9 & $58.8^{b}$ & $53.2^{c}$ & $45.7^{d}$ \\
Toileting hygiene & 63.9 & $52.8^{b}$ & $46.9^{c}$ & $41.2^{d}$ \\
Composite self-care & 63.3 & $55.8^{b}$ & $51.0^{c}$ & $45.4^{d}$ \\
Mobility items & & & & \\
Sit to lying & 66.1 & $57.5^{b}$ & $47.9^{c}$ & 45.5 \\
Lying to sitting & 66.0 & $57.3^{b}$ & $47.8^{c}$ & 45.4 \\
Sit to stand & 62.5 & $52.5^{b}$ & $46.7^{c}$ & $45.0^{c}$ \\
Chair/Bed-to-chair transfer & 60.9 & $51.4^{b}$ & $47.3^{c}$ & 45.4 \\
Toilet transfer & 60.2 & $51.3^{b}$ & $48.3^{c}$ & $44.6^{d}$ \\
Composite mobility & 62.3 & $53.9^{b}$ & $48.5^{c}$ & $44.6^{d}$ \\
\hline
\end{tabular}

${ }^{a}$ By cognitive status at admission $(\mathrm{N}=246,395)$.

$b_{p}<.001$ for severe cognitive impairment (ref.: moderate).

$c_{p}<.001$ for moderate cognitive impairment (ref.: mild).

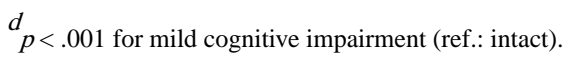

Notes: Means of the percentage of residents whose observed scores were the same Cognitive Function Scale (CFS)or higher than expected scores for all items were compared across groups of who were significantly different for all items as determined by analysis of variance $(p<.001)$. The $t$ tests were used to compare means of items between groups of CFS. 